Research Article

\title{
Simulation of the Final Size of the Evolution Curve of Coronavirus Epidemic in Morocco using the SIR Model
}

\author{
Ousama Ifguis, ${ }^{1}$ Mohamed El Ghozlani $\mathbb{D}^{2}{ }^{2}$ Fouzia Ammou, ${ }^{3}$ Abdelaziz Moutcine, ${ }^{4}$ \\ and Zeroual Abdellah ${ }^{5}$ \\ ${ }^{1}$ Laboratory of Chemical Processes and Applied Materials, Faculté des Sciences et Techniques, Université Sultan Moulay Slimane, \\ B.P. 523, Beni-Mellal, Morocco \\ ${ }^{2}$ Laboratoire de Chimie Organique et Analytiques, Faculté des Sciences et Techniques, Université Sultan Moulay Slimane, \\ B.P. 523, Beni-Mellal, Morocco \\ ${ }^{3}$ National Higher School of Electricity and Mechanics, Casablanca, Morocco \\ ${ }^{4}$ Electrochemistry and Molecular Inorganic Materials Team, Sultan Moulay Slimane University, \\ Faculty of Sciences and Technology, Beni-Mellal, Morocco \\ ${ }^{5}$ Molecular Modeling and Spectroscopy Research Team, Faculty of Science, Chouaïb Doukkali University, P.O. Box 20, \\ 24000 El Jadida, Morocco
}

Correspondence should be addressed to Mohamed El Ghozlani; elghozlanimohamed@gmail.com

Received 10 April 2020; Accepted 16 May 2020; Published 2 June 2020

Academic Editor: Chunrong Jia

Copyright (c) 2020 Ousama Ifguis et al. This is an open access article distributed under the Creative Commons Attribution License, which permits unrestricted use, distribution, and reproduction in any medium, provided the original work is properly cited.

Since the epidemic of COVID-19 was declared in Wuhan, Hubei Province of China, and other parts of the world, several studies have been carried out over several regions to observe the development of the epidemic, to predict its duration, and to estimate its final size, using complex models such as the SEIR model or the simpler ones such as the SIR model. These studies showed that the SIR model is much more efficient than the SEIR model; therefore, we are applying this model in the Kingdom of Morocco since the appearance of the first case on 2 March 2020, with the objective of predicting the final size of the epidemic.

\section{Introduction}

During Christmas 2019, COVID-19 caused an epidemic in the city of Wuhan, Hubei Province of China [1]. It spread to the other parts of China and subsequently to many other countries around the world. Morocco is one of the countries affected by COVID-19. On 2 March 2020, the country identified the first case [2, 3], and as of 30 March 2020, confirmed cases reached up to 516 with a death toll of 27 [4]. Also, as the number of infected cases is increasing, it is necessary for modellers to estimate the severity of the epidemic in terms of the total number of people infected, the total number of confirmed cases, the total number of deaths, and basic reproduction and to predict the duration of the epidemic, the arrival of its peak, and its final size. This information can help public health agencies make informed decisions.
In this work, we used the SIR model to predict the development of the epidemic in the Kingdom of Morocco from the identification of the first case on 2 March 2020 in the city of Casablanca [2], given the reliability of the data and the definition of confirmed cases during this period and the simplicity of our forecasts and analyses. We were able to determine the detailed results of the SIR model calibration and the predictions of our model, including the distribution of the peak period, the prediction interval of future confirmed cases, and the total number of infected persons.

\section{The SIR Model}

In the SIR model, compartment $S$ refers to the sensitive population in Morocco, $I$ refers to the infectious population, and $R$ refers to confirmed cases [5]. The latency of COVID19 infection is biologically realistic due to an incubation 


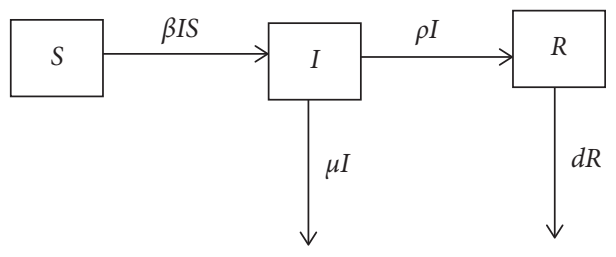

An SIR model

FIgUre 1: Transfer diagrams for the SIR model for COVID-19 in Morocco.

TABLE 1: Daily increased number of COVID-19 cases in Morocco [2].

\begin{tabular}{|c|c|c|c|c|}
\hline Date & Positive cases & Death cases & Reestablished cases & New cases \\
\hline 2 March 2020 & 1 & 0 & 0 & 1 \\
\hline 3 March 2020 & 1 & 0 & 0 & 0 \\
\hline 4 March 2020 & 1 & 0 & 0 & 0 \\
\hline 5 March 2020 & 2 & 0 & 0 & 1 \\
\hline 6 March 2020 & 2 & 0 & 0 & 0 \\
\hline 7 March 2020 & 2 & 0 & 0 & 0 \\
\hline 8 March 2020 & 2 & 0 & 0 & 0 \\
\hline 9 March 2020 & 2 & 0 & 0 & 0 \\
\hline 10 March 2020 & 3 & 1 & 0 & 1 \\
\hline 11 March 2020 & 6 & 1 & 0 & 3 \\
\hline 12 March 2020 & 6 & 1 & 1 & 0 \\
\hline 13 March 2020 & 8 & 1 & 1 & 2 \\
\hline 14 March 2020 & 18 & 1 & 1 & 10 \\
\hline 15 March 2020 & 28 & 1 & 1 & 10 \\
\hline 16 March 2020 & 37 & 1 & 1 & 9 \\
\hline 17 March 2020 & 44 & 2 & 1 & 7 \\
\hline 18 March 2020 & 54 & 2 & 1 & 10 \\
\hline 19 March 2020 & 63 & 2 & 2 & 9 \\
\hline 20 March 2020 & 86 & 3 & 2 & 23 \\
\hline 21 March 2020 & 96 & 3 & 3 & 10 \\
\hline 22 March 2020 & 115 & 4 & 3 & 19 \\
\hline 23 March 2020 & 143 & 4 & 5 & 28 \\
\hline 24 March 2020 & 170 & 5 & 6 & 27 \\
\hline 25 March 2020 & 225 & 6 & 7 & 55 \\
\hline 26 March 2020 & 275 & 10 & 8 & 50 \\
\hline 27 March 2020 & 345 & 23 & 11 & 70 \\
\hline 28 March 2020 & 402 & 25 & 12 & 57 \\
\hline 29 March 2020 & 479 & 26 & 13 & 77 \\
\hline 30 March 2020 & 556 & 33 & 15 & 77 \\
\hline 31 March 2020 & 617 & 36 & 24 & 61 \\
\hline
\end{tabular}

period of up to 14 days; newly infected persons may not be contagious during this time period as the virus will be incubating in the organism.

Here, we highlight the difference between the latency period, the period between when an individual is infected and when he or she is infectious, and the incubation period, the period between when an individual is infected and when clinical symptoms, including fever and cough symptomatic of COVID-19, appear.

The transfer diagram for the model is shown in Figure 1. The biological significance of all model parameters is given in Table 1. A key assumption in both models is that deaths occurring in compartments $S, E$, and $I$ are negligible during the model prediction period. The differential equation system for the SIR model is given as follows [5]:

$$
\begin{aligned}
& \frac{\mathrm{d} S}{\mathrm{~d} t}=-\beta S I, \\
& \frac{\mathrm{d} I}{\mathrm{~d} t}=\beta S I-\gamma I, \\
& \frac{\mathrm{d} I}{\mathrm{~d} t}=\gamma I .
\end{aligned}
$$

We can estimate the nature of the disease in terms of the power of infection:

$$
R_{0}=\frac{\beta}{\gamma} .
$$


TABLE 2: The estimation of parameters of the SIR model.

\begin{tabular}{|c|c|c|c|c|c|c|c|}
\hline Country & $\begin{array}{l}\text { Initial number of cases } \\
\qquad\left(I_{0}\right)\end{array}$ & $\begin{array}{c}\text { Contact rate } \\
(\beta)\end{array}$ & $\begin{array}{l}\text { Removal } \\
\text { rate }(\gamma)\end{array}$ & $\begin{array}{c}\text { Basic } \\
\text { reproduction } \\
\text { number }\left(R_{0}\right)\end{array}$ & $\begin{array}{c}\text { Critical number } \\
\text { of susceptible } \\
\text { cases }\end{array}$ & $\begin{array}{c}\text { Final } \\
\text { number } \\
\text { of cases }\end{array}$ & $\begin{array}{c}\text { Final number of } \\
\text { susceptible } \\
\text { cases }\end{array}$ \\
\hline Morocco & 1 & $\begin{array}{c}0.499 \\
\text { (1/day) }\end{array}$ & $\begin{array}{c}0.249 \\
\text { (1/day) }\end{array}$ & 2.003 & 1,812 & 1,446 & 366 \\
\hline
\end{tabular}

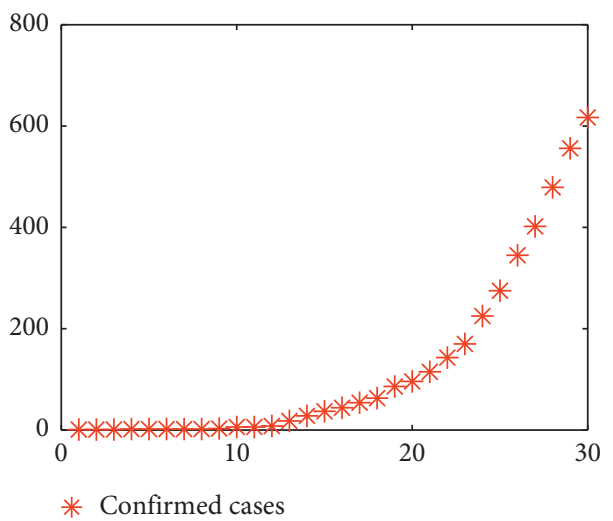

(a)

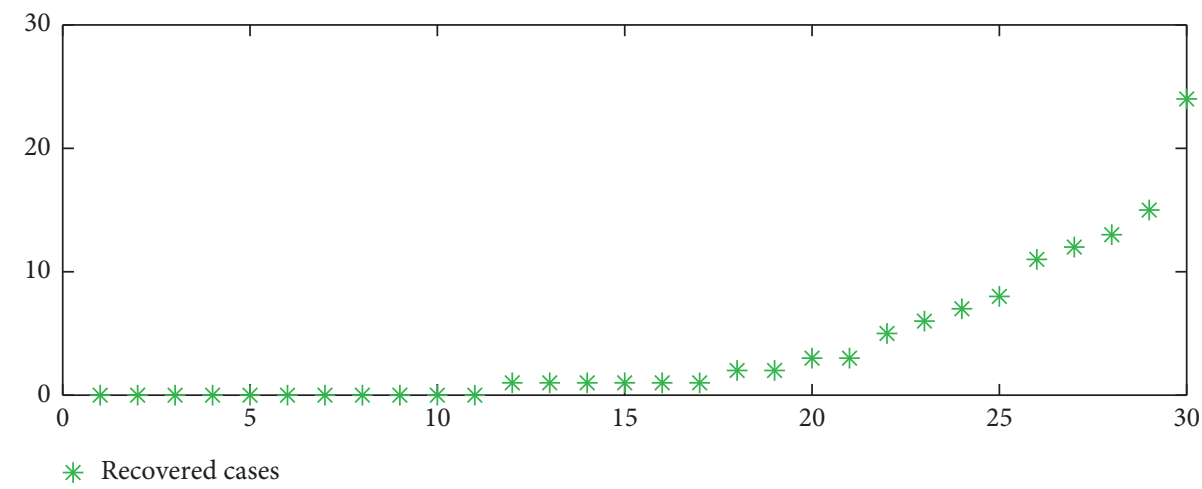

(c)

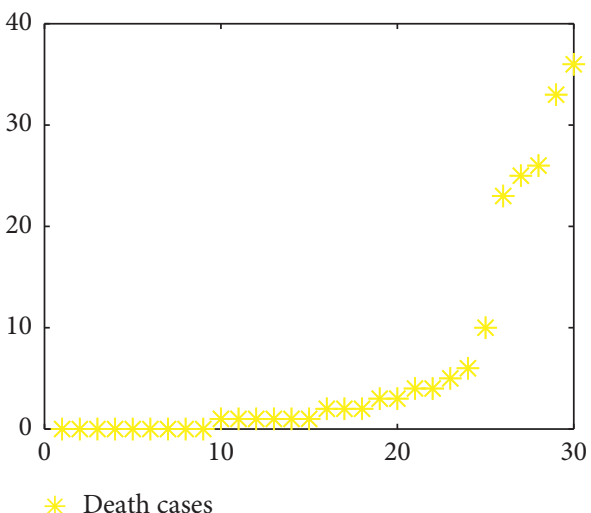

(b) 30

Figure 2: (a) Confirmed cases, (b) death cases, and (c) recovered cases from 2 March 2020 to 31 March 2020.

It is called the basic reproduction number. $R_{0}$ is the average number of people infected from another person. If it is high, the probability of the pandemic is also higher.

\section{Simulation with COVID-19 Data in Morocco}

For this study, we used the data accumulated since 2 March 2020 concerning Morocco [2]. Four parameters to be estimated in the SIR model from the data are as follows: basic reproduction number $\left(R_{0}\right)$, contact rate $(\beta)$, removal rate $(\gamma)$, and final number of cases $(I)$.

We do not consider the effect of the natural death or birth rate so that the total population remains constant.
(i) $N=$ constant
(ii) $N_{0}=$ total population in Morocco $=40,000,000$
(iii) $I_{0}=1$
(iv) $R_{0}=0$

\section{Results}

To solve the ordinary differential equation of the SIR model (equations (1)-(3)), we must first simulate the daily data of the SIR model, compare them with the real data, and then execute the optimization algorithm (reduce the difference between the real data and the corresponding simulated data), so that it searches for the values of $\alpha$ and $\beta$, which minimize the difference between the real data and the simulated ones.

In the optimal model, we supposed that the predicted infected cases should definitely be close to the actual number of infected cases; we found that the critical number of susceptible cases was about 1,812 (Table 2).

The transfer diagram for the SIR model used in the simulation of the size of COVID-19 infection in the Moroccan state is provided in detail in Figure 1.

We note from Figure 2 that the number of confirmed cases is not very high; the highest daily confirmed number 


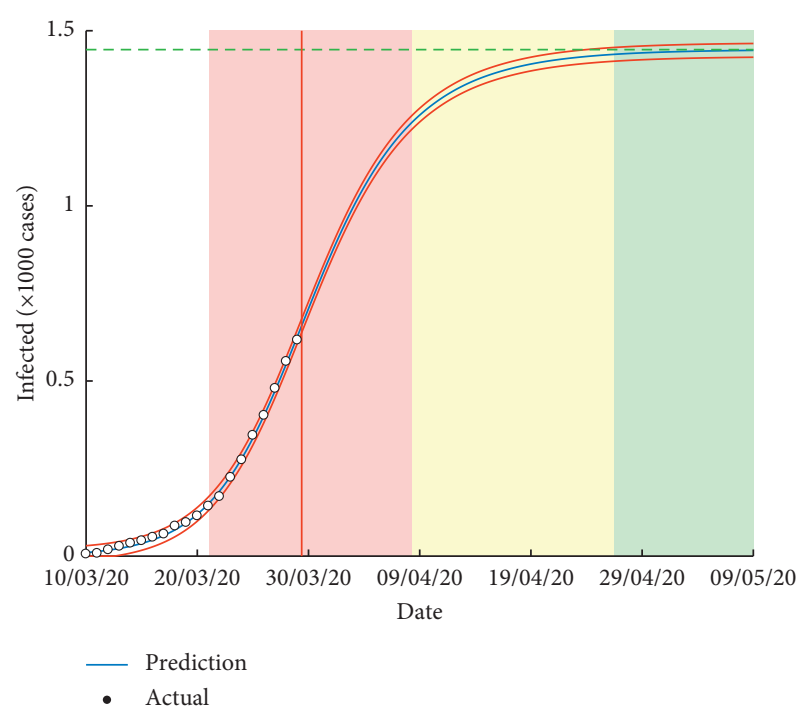

Figure 3: Presentation of the evolution of total infected cases $\left(R_{0}=2.003, \beta=0.499, \gamma=0.249, N=1,812, C_{\text {end }}=1,446, S_{\text {end }}=366\right.$, RMSE $=7$ ).

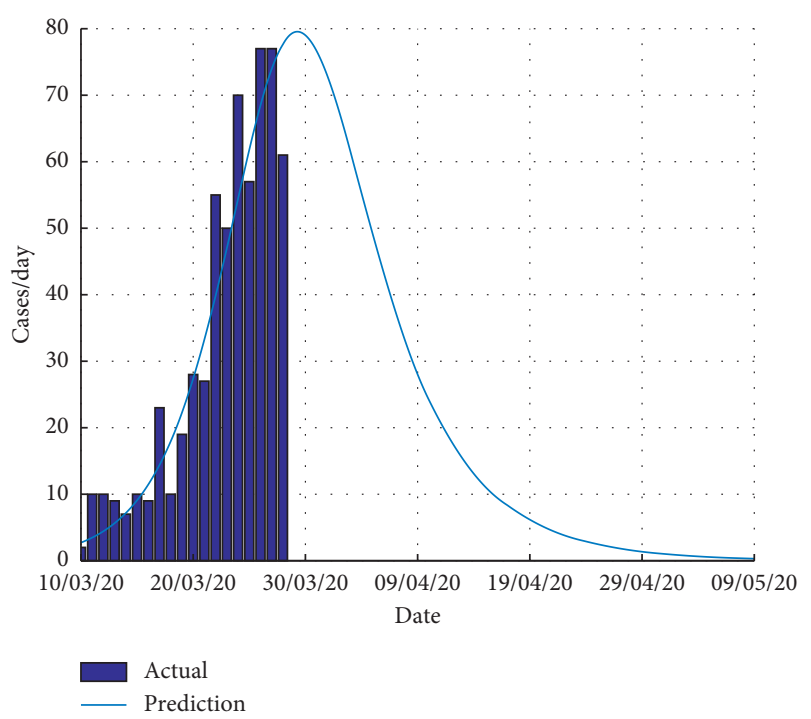

FIgURE 4: Daily estimated number of reported cases.

TABLE 3: Basic reproduction number $R_{0}(t)$ of the time-dependent SIR model of COVID-19 in Morocco based on the given data from 21 March 2020 to 31 March 2020.

\begin{tabular}{lc}
\hline Days & The reproduction number $R_{0}$ \\
\hline 21 March 2020 & 1,751 \\
22 March 2020 & 149 \\
23 March 2020 & 1,182 \\
24 March 2020 & 1,229 \\
25 March 2020 & 1,339 \\
26 March 2020 & 1,586 \\
27 March 2020 & 1,337 \\
28 March 2020 & 2,559 \\
\hline
\end{tabular}

was 70 on 28 March 2020. This can be explained by the strategy of Morocco, which declared a state of health emergency and confinement on 20 March 2020, Friday, at 6 p.m., to limit the displacement of the population as much as possible, the only way to keep the coronavirus under control.

Figure 3, based on optimal SIR models, shows that the start of acceleration of the epidemic is around 21 March 2020, the regular growth will begin on 8 April 2020, and the end of the epidemic in Morocco would be around 26 April 2020, with a total of 1,446 infected cases and 366 final number of susceptible cases (Table 2).

The peak of the reported cases will be around 28 March 2020 with 80 confirmed cases (Figure 4).

The optimal estimates of $R_{0}$ ranges from 1.22 to 2.55 (Table 3); our study confirmed that the transmissibility of COVID-19 is $R_{0}=2.003$ (Table 3 ), which was comparable to that of severe acute respiratory syndrome coronavirus (SARSCoV) (ranging from 2.2 to 3.7) [6] and much higher than that of Middle East respiratory syndrome coronavirus (MERS-CoV) (ranging from 0.47 to 0.91) [7].

\section{Conclusion}

Our simulation study on the optimization of the final size of COVID-19 epidemic evolution in the Kingdom of Morocco, with the SIR model, has allowed us to accurately predict the peak of the infected and death cases (Table 2), although the number of people tested is very low, about 3,079, until 31 March 2020.

The Moroccan government should probably increase the number of cases tested on a daily basis in order to accurately identify the true size of the pandemic in Morocco.

\section{Data Availability}

The data used to support the findings of this study are cited in the article as Reference [3]. And the mathematical calculations are provided in the Supplementary Materials.

\section{Conflicts of Interest}

The authors declare that they have no conflicts of interest.

\section{Supplementary Materials}

The mathematical calculations and also the different program steps to obtain the graphs are provided. (Supplementary Materials)

\section{References}

[1] N. P. A. S. Johnson and J. Mueller, "Updating the accounts: global mortality of the 1918-1920 "Spanish" influenza pandemic," Bulletin of the History of Medicine, vol. 76, no. 1, pp. 105-115, 2002.

[2] W. O. Kermack and A. G. McKendrick, A Contribution to the Mathematical Theory of Epidemics, Vol. 115, Proceedings of the Royal Society of London: Series A, London, UK, 1927. 
[3] World Health Organization, Coronavirus Disease 2019 (COVID-19): Situation Report, 42, WHO, Geneva, Switzerland, 2020.

[4] World Health Organization, Coronavirus Disease 2019 (COVID-19): Situation Report, 70, WHO, Geneva, Switzerland, 2020.

[5] W. C. Roda, M. B. Varughese, D. Han, and M. Y. Li, "Why is it difficult to accurately predict the COVID-19 epidemic?" Infectious Disease Modelling, vol. 5, pp. 271-281, 2020.

[6] S. Riley, C. Fraser, C. A. Donnelly et al., "Transmission dynamics of the etiological agent of SARS in Hong Kong: impact of public health interventions," Science, vol. 300, no. 5627, pp. 1961-1966, 2003.

[7] S. Cauchemez, P. Nouvellet, A. Cori et al., "Unraveling the drivers of MERS-CoV transmission," Proceedings of the $\mathrm{Na}$ tional Academy of Sciences, vol. 113, no. 32, pp. 9081-9086, 2016. 